\title{
Scaled tree fractals do not strictly self-assemble
}

\author{
Kimberly Barth ${ }^{\star}$, David Furcy ${ }^{\star \star}$, Scott M. Summers ${ }^{\star \star \star}$, and Paul Totzke ${ }^{\dagger}$
}

\begin{abstract}
In this paper, we show that any scaled-up version of any discrete self-similar tree fractal does not strictly self-assemble, at any temperature, in Winfree's abstract Tile Assembly Model.
\end{abstract}

\section{Introduction}

The stunning, often mysterious complexities of the natural world, from nanoscale crystalline structures to unthinkably massive galaxies, all arise from the same elemental process known as self-assembly. In the absence of a mathematically rigorous definition, self-assembly is colloquially thought of as the process through which simple, unorganized components spontaneously combine, according to local interaction rules, to form some kind of organized final structure. A major objective of nanotechnology is to harness the power of self-assembly, perhaps for the purpose of engineering atomically precise medical, digital and mechanical components at the nanoscale. One strategy for doing so, developed by Nadrian Seeman, is DNA tile self-assembly [8,9].

In DNA tile self-assembly, the fundamental components are "tiles", which are comprised of interconnected DNA strands. Remarkably, these DNA tiles can be "programmed", via the careful configuration of their constituent DNA strands, to automatically coalesce into a desired target structure, the characteristics of which are completely determined by the "programming" of the DNA tiles. In order to fully realize the power of DNA tile self-assembly, we must study the algorithmic and mathematical underpinnings of tile self-assembly.

Perhaps the simplest mathematical model of algorithmic tile self-assembly is Erik Winfree's abstract Tile Assembly Model (aTAM) [11. The aTAM is a deliberately over-simplified, combinatorial model of nanoscale (DNA) tile selfassembly that "effectivizes" classical Wang tiling [10] in the sense that the former augments the latter with a mechanism for sequential "growth" of a tile assembly. Very briefly, in the aTAM, the fundamental components are un-rotatable, translatable square "tile types" whose sides are labeled with (alpha-numeric) glue "colors" and (integer) "strengths". Two tiles that are placed next to each other

\footnotetext{
* Department of Computer Science, University of Wisconsin-Oshkosh, Oshkosh, WI 54901, USA, barthk63@uwosh.edu.

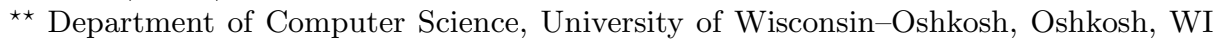
54901, USA, furcyd@uwosh.edu.

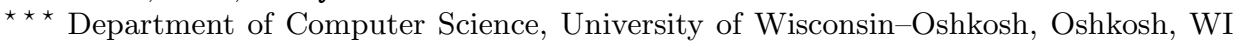
54901, USA, summerss@uwosh.edu.

$\dagger$ Department of Computer Science, University of Wisconsin-Oshkosh, Oshkosh, WI 54901, USA, totzkp00@uwosh.edu.
} 
interact if the glue colors on their abutting sides match, and they bind if the strengths on their abutting sides match and sum to at least a certain (integer) "temperature". Self-assembly starts from a "seed" tile type, typically assumed to be placed at the origin, and proceeds nondeterministically and asynchronously as tiles bind to the seed-containing assembly one at a time.

Despite its deliberate over-simplification, the aTAM is a computationally expressive model. For example, Winfree [11] proved that the model is Turing universal, which means that, in principle, the process of self-assembly can be directed by any algorithm. In this paper, we will specifically study the extent to which tile sets in the aTAM can be algorithmically directed to "strictly" selfassemble (place tiles at and only at locations that belong to) shapes that are discrete self-similar tree fractals.

There are examples of prior results related to the self-assembly of fractals in the aTAM, in general 1, 2, 6, as well as the strict self-assembly of tree fractals in the aTAM, specifically 3,4. In fact, a notable example of the latter is 3, Theorem 3.2 of which bounds from below the size of the smallest tile set in which an arbitrary shape $X$ strictly self-assembles by the depth of $X$ 's largest finite sub-tree. Although not stated explicitly, an immediate corollary of Theorem 3.2 of $[3$ is that no tree fractals strictly self-assemble in the aTAM.

While the strict self-assembly of tree fractals in the aTAM is well-understood (via Theorem 3.2 of [3]), nothing is known about the strict self-assembly of "scaled-up" versions of tree fractals ("scaled-up" meaning each point in the original shape is replaced by a $c \times c$ block of points). After all, the scaled-up version of any shape - tree or otherwise - is not a tree in the sense of the "full connectivity graph" of the shape, i.e., each point in the shape is represented by one vertex and edges exist between vertices that represent adjacent points in the shape. This means that prior proof techniques, which exploit the intricate geometry of tree fractals (e.g., 3, 4), simply cannot be applied to scaled-up versions of tree fractals. Thus, in this paper, we ask if it is possible for a scaledup version of a tree fractal to strictly self-assemble in the aTAM.

The main contribution of this paper provides an answer to the previous question, perhaps not too surprisingly to readers familiar with the aTAM, in the negative: we prove that there is no tree fractal that strictly self-assembles in the aTAM - at any positive scale factor. Thus, our main result generalizes Theorem 3.4 of [3, which says that the Sierpinski triangle, perhaps the most famous, wellstudied example of a tree fractal, does not strictly self-assemble at scale factor 1 . Our proof makes crucial use of a recent technical lemma developed by Meunier, Patitz, Summers, Theyssier, Winslow and Woods [5, known as the "Window Movie Lemma" (WML), which gives a sufficient condition for taking any pair of tile assemblies, at any temperature, and "splicing" them together to create a new valid tile assembly. The WML is, in some sense, a pumping lemma for self-assembly that mitigates the need to use overly-complicated, convoluted caseanalyses that typically arise when doing impossibility proofs in self-assembly.

What follows is a list of the main technical contributions presented in - and the general outline of - this paper: 
- In Section 2.2, we exhibit a natural characterization of a tree fractal in terms of a few simple, easily checkable geometric properties of its generator. While perhaps well-known, this type of characterization, to the best of our knowledge, has yet to be explicitly documented or proved in the literature.

- In Section 2.3, we develop a modified version of the general WML. Our version of the WML, which we call the "Closed Window Movie Lemma", allows us to replace one portion of a tile assembly with another, assuming certain extra "containment" conditions are met. Moreover, unlike in the original WML that lacks the extra containment assumptions, the replacement of one tile assembly with another in our Closed WML only goes "one way", i.e., the part of the tile assembly being used to replace another part cannot itself be replaced by the part of the tile assembly it is replacing.

- In Section 3, we use our closed WML to prove that any scaled-up version of any tree fractal does not strictly self-assemble in the aTAM at any temperature. Our main result generalizes the claim that every tree fractal, at scale factor 1 , does not strictly self-assemble in the aTAM (an implicit corollary to the main negative result of [3]).

\section{Definitions}

In this section, we give a formal definition of Erik Winfree's abstract Tile Assembly Model (aTAM), define and characterize tree fractals and develop a "Closed" Window Movie Lemma.

\subsection{Formal description of the abstract Tile Assembly Model}

This section gives a formal definition of the abstract Tile Assembly Model (aTAM) [11. For readers unfamiliar with the aTAM, 7] gives an excellent introduction to the model.

Fix an alphabet $\Sigma$. $\Sigma^{*}$ is the set of finite strings over $\Sigma$. Let $\mathbb{Z}, \mathbb{Z}^{+}$, and $\mathbb{N}$ denote the set of integers, positive integers, and nonnegative integers, respectively. Given $V \subseteq \mathbb{Z}^{2}$, the full grid graph of $V$ is the undirected graph $G_{V}^{\mathrm{f}}=(V, E)$, such that, for all $\boldsymbol{x}, \boldsymbol{y} \in V,\{\boldsymbol{x}, \boldsymbol{y}\} \in E \Longleftrightarrow\|\boldsymbol{x}-\boldsymbol{y}\|=1$, i.e., if and only if $\boldsymbol{x}$ and $\boldsymbol{y}$ are adjacent in the 2-dimensional integer Cartesian space.

A tile type is a tuple $t \in\left(\Sigma^{*} \times \mathbb{N}\right)^{4}$, e.g., a unit square, with four sides, listed in some standardized order, and each side having a glue $g \in \Sigma^{*} \times \mathbb{N}$ consisting of a finite string label and a nonnegative integer strength.

We assume a finite set of tile types, but an infinite number of copies of each tile type, each copy referred to as a tile. A tile set is a set of tile types and is usually denoted as $T$.

A configuration is a (possibly empty) arrangement of tiles on the integer lattice $\mathbb{Z}^{2}$, i.e., a partial function $\alpha: \mathbb{Z}^{2} \rightarrow T$. Two adjacent tiles in a configuration interact, or are attached, if the glues on their abutting sides are equal (in both label and strength) and have positive strength. Each configuration $\alpha$ induces a binding graph $G_{\alpha}^{\mathrm{b}}$, a grid graph whose vertices are positions occupied by tiles, 
according to $\alpha$, with an edge between two vertices if the tiles at those vertices interact. An assembly is a connected, non-empty configuration, i.e., a partial function $\alpha: \mathbb{Z}^{2} \rightarrow T$ such that $G_{\mathrm{dom} \alpha}^{\mathrm{f}}$ is connected and dom $\alpha \neq \varnothing$.

Given $\tau \in \mathbb{Z}^{+}, \alpha$ is $\tau$-stable if every cut-set of $G_{\alpha}^{\mathrm{b}}$ has weight at least $\tau$, where the weight of an edge is the strength of the glue it represents 1 When $\tau$ is clear from context, we say $\alpha$ is stable. Given two assemblies $\alpha, \beta$, we say $\alpha$ is a subassembly of $\beta$, and we write $\alpha \sqsubseteq \beta$, if $\operatorname{dom} \alpha \subseteq \operatorname{dom} \beta$ and, for all points $p \in \operatorname{dom} \alpha, \alpha(p)=\beta(p)$. For two non-overlapping assemblies $\alpha$ and $\beta, \alpha \cup \beta$ is defined as the unique assembly $\gamma$ satisfying, for all $\boldsymbol{x} \in \operatorname{dom} \alpha$, $\gamma(\boldsymbol{x})=\alpha(\boldsymbol{x})$, for all $\boldsymbol{x} \in \operatorname{dom} \beta, \gamma(\boldsymbol{x})=\beta(\boldsymbol{x})$, and $\gamma(\boldsymbol{x})$ is undefined at any point $\boldsymbol{x} \in \mathbb{Z}^{2} \backslash(\operatorname{dom} \alpha \cup \operatorname{dom} \beta)$.

A tile assembly system (TAS) is a triple $\mathcal{T}=(T, \sigma, \tau)$, where $T$ is a tile set, $\sigma: \mathbb{Z}^{2} \rightarrow T$ is the finite, $\tau$-stable, seed assembly, and $\tau \in \mathbb{Z}^{+}$is the temperature.

Given two $\tau$-stable assemblies $\alpha, \beta$, we write $\alpha \rightarrow_{1}^{\mathcal{T}} \beta$ if $\alpha \sqsubseteq \beta$ and $\mid \operatorname{dom} \beta \backslash$ $\operatorname{dom} \alpha \mid=1$. In this case we say $\alpha \mathcal{T}$-produces $\beta$ in one step. If $\alpha \rightarrow_{1}^{\mathcal{T}} \beta$, $\operatorname{dom} \beta \backslash \operatorname{dom} \alpha=\{p\}$, and $t=\beta(p)$, we write $\beta=\alpha+(p \mapsto t)$. The $\mathcal{T}$-frontier of $\alpha$ is the set $\partial^{\mathcal{T}} \alpha=\bigcup_{\alpha \rightarrow{ }_{1} \beta}(\operatorname{dom} \beta \backslash \operatorname{dom} \alpha)$, the set of empty locations at which a tile could stably attach to $\alpha$. The $t$-frontier $\partial_{t}^{\mathcal{T}} \alpha \subseteq \partial^{\mathcal{T}} \alpha$ of $\alpha$ is the set $\left\{p \in \partial^{\mathcal{T}} \alpha \mid \alpha \rightarrow_{1}^{\mathcal{T}} \beta\right.$ and $\left.\beta(p)=t\right\}$.

Let $\mathcal{A}^{T}$ denote the set of all assemblies of tiles from $T$, and let $\mathcal{A}_{<\infty}^{T}$ denote the set of finite assemblies of tiles from $T$. A sequence of $k \in \mathbb{Z}^{+} \cup\{\infty\}$ assemblies $\alpha_{0}, \alpha_{1}, \ldots$ over $\mathcal{A}^{T}$ is a $\mathcal{T}$-assembly sequence if, for all $1 \leq i<k, \alpha_{i-1} \rightarrow_{1}^{\mathcal{T}} \alpha_{i}$. The result of an assembly sequence $\boldsymbol{\alpha}$, denoted as res $(\boldsymbol{\alpha})$, is the unique limiting assembly (for a finite sequence, this is the final assembly in the sequence).

We write $\alpha \rightarrow^{\mathcal{T}} \beta$, and we say $\alpha \mathcal{T}$-produces $\beta$ (in 0 or more steps) if there is a $\mathcal{T}$-assembly sequence $\alpha_{0}, \alpha_{1}, \ldots$ of length $k=|\operatorname{dom} \beta \backslash \operatorname{dom} \alpha|+1$ such that (1) $\alpha=\alpha_{0},(2) \operatorname{dom} \beta=\bigcup_{0 \leq i<k} \operatorname{dom} \alpha_{i}$, and (3) for all $0 \leq i<k, \alpha_{i} \sqsubseteq \beta$. If $k$ is finite then it is routine to verify that $\beta=\alpha_{k-1}$.

We say $\alpha$ is $\mathcal{T}$-producible if $\sigma \rightarrow^{\mathcal{T}} \alpha$, and we write $\mathcal{A}[\mathcal{T}]$ to denote the set of $\mathcal{T}$-producible assemblies. The relation $\rightarrow^{\mathcal{T}}$ is a partial order on $\mathcal{A}[\mathcal{T}]$ [3,7].

An assembly $\alpha$ is $\mathcal{T}$-terminal if $\alpha$ is $\tau$-stable and $\partial^{\mathcal{T}} \alpha=\varnothing$. We write $\mathcal{A}_{\square}[\mathcal{T}] \subseteq$ $\mathcal{A}[\mathcal{T}]$ to denote the set of $\mathcal{T}$-producible, $\mathcal{T}$-terminal assemblies. If $\left|\mathcal{A}_{\square}[\mathcal{T}]\right|=1$ then $\mathcal{T}$ is said to be directed.

We say that a TAS $\mathcal{T}$ strictly (a.k.a. uniquely) self-assembles $X \subseteq \mathbb{Z}^{2}$ if, for all $\alpha \in \mathcal{A}_{\square}[\mathcal{T}]$, dom $\alpha=X$; i.e., if every terminal assembly produced by $\mathcal{T}$ places tiles on - and only on - points in the set $X$.

In this paper, we consider scaled-up versions of subsets of $\mathbb{Z}^{2}$. Formally, if $X$ is a subset of $\mathbb{Z}^{2}$ and $c \in \mathbb{Z}^{+}$, then a c-scaling of $X$ is defined as the set $X^{c}=\left\{(x, y) \in \mathbb{Z}^{2} \mid\left(\left\lfloor\frac{x}{c}\right\rfloor,\left\lfloor\frac{y}{c}\right\rfloor\right) \in X\right\}$. Intuitively, $X^{c}$ is the subset of $\mathbb{Z}^{2}$ obtained by replacing each point in $X$ with a $c \times c$ block of points. We refer to the natural number $c$ as the scaling factor or resolution loss.

\footnotetext{
${ }^{1}$ A cut-set is a subset of edges in a graph which, when removed from the graph, produces two or more disconnected subgraphs. The weight of a cut-set is the sum of the weights of all of the edges in the cut-set.
} 


\subsection{Discrete self-similar tree fractals}

In this section, we introduce a new formal characterization of discrete self-similar tree fractals. The proof of Theorem 1 below is omitted from this version of the paper due to lack of space.

Notation 1 We use $\mathbb{N}_{g}$ to denote the subset $\{0, \ldots, g-1\}$ of $\mathbb{N}$.

Notation 2 If $A$ and $B$ are subsets of $\mathbb{N}^{2}$ and $k \in \mathbb{N}$, then $A+k B=\{\boldsymbol{m}+$ $k \boldsymbol{n} \mid \boldsymbol{m} \in A$ and $\boldsymbol{n} \in B\}$.

The following definition is adapted from $[\underline{6}$.

Definition 1. Let $1<g \in \mathbb{N}$ and $\mathbf{X} \subset \mathbb{N}^{2}$. We say that $\mathbf{X}$ is a $g$-discrete selfsimilar fractal (or g-dssf for short), if there is a set $\{(0,0)\} \subset G \subset \mathbb{N}_{g}^{2}$ with at least one point in every row and column, such that $\mathbf{X}=\bigcup_{i=1}^{\infty} X_{i}$, where $X_{i}$, the $i^{\text {th }}$ stage of $\mathbf{X}$, is defined by $X_{1}=G$ and $X_{i+1}=X_{i}+g^{i} G$. We say that $G$ is the generator of $\mathbf{X}$.

Intuitively, a $g$-dssf is built as follows. Start by selecting points in $\mathbb{N}_{g}^{2}$ satisfying the constraints listed in Definition [1 This first stage of the fractal is the generator. Then, each subsequent stage of the fractal is obtained by adding a full copy of the previous stage for every point in the generator and translating these copies so that their relative positions are identical to the relative positions of the individual points in the gnerator.

In this paper, we focus on tree fractals, that is, fractals whose underlying graph is a tree. We introduce terminology and notation that will help us in formulating a complete characterization of tree fractals in terms of geometric properties of their generator.

Definition 2. Let $S$ be any finite subset of $\mathbb{Z}^{2}$. Let $l, r, b$, and $t$ denote the following integers:

$$
l_{S}=\min _{(x, y) \in S} x \quad r_{S}=\max _{(x, y) \in S} x \quad b_{S}=\min _{(x, y) \in S} y \quad t_{S}=\max _{(x, y) \in S} y
$$

An h-bridge of $S$ is any subset of $S$ of the form $h b_{S}(y)=\left\{\left(l_{S}, y\right),\left(r_{S}, y\right)\right\}$. Similarly, a v-bridge of $S$ is any subset of $S$ of the form $v b_{S}(x)=$ $\left\{\left(x, b_{S}\right),\left(x, t_{S}\right)\right\}$. We say that a bridge is connected if there is a simple path in $S$ connecting the two bridge points.

Notation 3 Let $S$ be any finite subset of $\mathbb{Z}^{2}$. We will denote by $n h b_{S}$ and $n v b_{S}$, respectively, the number of $h$-bridges and the number of v-bridges of $S$.

The following theorem is a new characterization of tree fractals in terms of simple connectivity properties of their generator. 
Theorem 1. $\mathbf{T}=\bigcup_{i=1}^{\infty} T_{i}$ is a $g$-discrete self-similar tree fractal, for some $g>1$, with generator $G$ if and only if

a. $G$ is a tree, and

b. $n h b_{G}=n v b_{G}=1$

Notation 4 The directions $\mathcal{D}=\{N, E, S, W\}$ will be used as functions from $\mathbb{Z}^{2}$ to $\mathbb{Z}^{2}$ such that $N(x, y)=(x, y+1), E(x, y)=(x+1, y), S(x, y)=(x, y-1)$ and $W(x, y)=(x-1, y)$. Note that $N^{-1}=S$ and $W^{-1}=E$.

Notation 5 Let $X \subseteq \mathbb{Z}^{2}$. We say that a point $(x, y) \in X$ is $D$-free in $X$, for some direction $D$, if $D(x, y) \notin X$.

Definition 3. Let $G$ be the generator of any g-discrete self-similar fractal. A pier is a point in $G$ that is $D$-free for exactly three of the four directions in $\mathcal{D}$. We say that a pier $(p, q)$ is $D$-pointing if $D^{-1}(p, q) \in G$. Note that a pier always points in exactly one direction

Finally, the following observation follows from the fact that a tree with more than one vertex must contain at least two leaf nodes.

Observation 1 If $G$ is the generator of any discrete self-similar fractal and $G$ is a tree, then it must contain at least two piers.

\subsection{The Closed Window Movie Lemma}

In this subsection, we develop a more accommodating (modified) version of the general Window Movie Lemma (WML) [5. Our version of the WML, which we call the "Closed Window Movie Lemma", allows us to replace one portion of a tile assembly with another, assuming certain extra "containment" conditions are met. Moreover, unlike in the original WML that lacks the extra containment assumptions, the replacement of one tile assembly with another in our Closed WML only goes "one way", i.e., the part of the tile assembly being used to replace another part cannot itself be replaced by the part of the tile assembly it is replacing. We must first define some notation that we will use in our closed Window Movie Lemma.

A window $w$ is a set of edges forming a cut-set of the full grid graph of $\mathbb{Z}^{2}$. For the purposes of this paper, we say that a closed window $w$ induces a cut 2 of the full grid graph of $\mathbb{Z}^{2}$, written as $C_{w}=\left(C_{<\infty}, C_{\infty}\right)$, where $C_{\infty}$ is infinite, $C_{<\infty}$ is finite and for all pairs of points $\boldsymbol{x}, \boldsymbol{y} \in C_{<\infty}$, every simple path connecting $\boldsymbol{x}$ and $\boldsymbol{y}$ in the full grid graph of $C_{<\infty}$ does not cross the cut $C_{w}$. We call the set of vertices that make up $C_{<\infty}$ the inside of the window $w$, and write $\operatorname{inside}(w)=C_{<\infty}$ and outside $(w)=\mathbb{Z}^{2} \backslash \operatorname{inside}(w)=C_{\infty}$. We say that a window $w$ is enclosed in another window $w^{\prime}$ if inside $(w) \subseteq$ inside $\left(w^{\prime}\right)$.

${ }^{2}$ A cut is a partition of the vertices of a graph into two disjoint subsets that are joined by at least one edge. 
Given a window $w$ and an assembly $\alpha$, a window that intersects $\alpha$ is a partitioning of $\alpha$ into two configurations (i.e., after being split into two parts, each part may or may not be disconnected). In this case we say that the window $w$ cuts the assembly $\alpha$ into two configurations $\alpha_{L}$ and $\alpha_{R}$, where $\alpha=\alpha_{L} \cup \alpha_{R}$. For notational convenience, if $w$ is a closed window, we write $\alpha_{I}$ for the assembly inside $w$ and $\alpha_{O}$ for the assembly outside $w$. Given a window $w$, its translation by a vector $\boldsymbol{c}$, written $w+c$ is simply the translation of each of $w$ 's elements (edges) by $\boldsymbol{c}$.

For a window $w$ and an assembly sequence $\boldsymbol{\alpha}$, we define a window movie $M$ to be the order of placement, position and glue type for each glue that appears along the window $w$ in $\boldsymbol{\alpha}$. Given an assembly sequence $\boldsymbol{\alpha}$ and a window $w$, the associated window movie is the maximal sequence $M_{\boldsymbol{\alpha}, w}=\left(v_{0}, g_{0}\right),\left(v_{1}, g_{1}\right),\left(v_{2}, g_{2}\right), \ldots$ of pairs of grid graph vertices $v_{i}$ and glues $g_{i}$, given by the order of the appearance of the glues along window $w$ in the assembly sequence $\boldsymbol{\alpha}$. Furthermore, if $k$ glues appear along $w$ at the same instant (this happens upon placement of a tile that has multiple sides touching $w$ ) then these $k$ glues appear contiguously and are listed in lexicographical order of the unit vectors describing their orientation in $M_{\boldsymbol{\alpha}, w}$.

Let $w$ be a window and $\boldsymbol{\alpha}$ be an assembly sequence and $M=M_{\boldsymbol{\alpha}, w}$. We use the notation $\mathcal{B}(M)$ to denote the bond-forming submovie of $M$, i.e., a restricted form of $M$ that consists of only those steps of $M$ that place glues that eventually form positive-strength bonds in the assembly $\alpha=\operatorname{res}(\boldsymbol{\alpha})$. Note that every window movie has a unique bond-forming submovie.

Lemma 1 (Closed Window Movie Lemma). Let $\boldsymbol{\alpha}=\left(\alpha_{i} \mid 0 \leq i<l\right)$, with $l \in \mathbb{Z}^{+} \cup\{\infty\}$, be an assembly sequence in some TAS $\mathcal{T}$ with result $\alpha$. Let $w$ be a closed window that partitions $\alpha$ into $\alpha_{I}$ and $\alpha_{O}$, and $w^{\prime}$ be a closed window that partitions $\alpha$ into $\alpha_{I}^{\prime}$ and $\alpha_{O}^{\prime}$. If $\mathcal{B}\left(M_{\boldsymbol{\alpha}, w}\right)+\boldsymbol{c}=\mathcal{B}\left(M_{\boldsymbol{\alpha}, w^{\prime}}\right)$ for some $\boldsymbol{c} \neq(0,0)$ and the window $w+\boldsymbol{c}$ is enclosed in $w^{\prime}$, then the assembly $\alpha_{O}^{\prime} \cup\left(\alpha_{I}+\boldsymbol{c}\right)$ is in $\mathcal{A}[\mathcal{T}]$.

Proof. Before we proceed with the proof, the next paragraph introduces some notation taken directly from [5].

For an assembly sequence $\boldsymbol{\alpha}=\left(\alpha_{i} \mid 0 \leq i<l\right)$, we write $|\boldsymbol{\alpha}|=l$ (note that if $\boldsymbol{\alpha}$ is infinite, then $l=\infty)$. We write $\boldsymbol{\alpha}[i]$ to denote $\boldsymbol{x} \mapsto t$, where $\boldsymbol{x}$ and $t$ are such that $\alpha_{i+1}=\alpha_{i}+(\boldsymbol{x} \mapsto t)$, i.e., $\boldsymbol{\alpha}[i]$ is the placement of tile type $t$ at position $\boldsymbol{x}$, assuming that $\boldsymbol{x} \in \partial_{t} \alpha_{i}$. We write $\boldsymbol{\alpha}[i]+\boldsymbol{c}$, for some vector $\boldsymbol{c}$, to denote $(\boldsymbol{x}+\boldsymbol{c}) \mapsto t$. We define $\boldsymbol{\alpha}=\boldsymbol{\alpha}+(\boldsymbol{x} \mapsto t)=\left(\alpha_{i} \mid 0 \leq i<k+1\right)$, where $\alpha_{k}=\alpha_{k-1}+(\boldsymbol{x} \mapsto t)$ if $\boldsymbol{x} \in \partial_{t} \alpha_{k-1}$ and undefined otherwise, assuming $|\boldsymbol{\alpha}|>0$. Otherwise, if $|\boldsymbol{\alpha}|=0$, then $\boldsymbol{\alpha}=\boldsymbol{\alpha}+(\boldsymbol{x} \mapsto t)=\left(\alpha_{0}\right)$, where $\alpha_{0}$ is the assembly such that $\alpha_{0}(\boldsymbol{x})=t$ and is undefined at all other positions. This is our notation for appending steps to the assembly sequence $\boldsymbol{\alpha}$ : to do so, we must specify a tile type $t$ to be placed at a given location $\boldsymbol{x} \in \partial_{t} \alpha_{i}$. If $\alpha_{i+1}=\alpha_{i}+(\boldsymbol{x} \mapsto t)$, then we write $\operatorname{Pos}(\boldsymbol{\alpha}[i])=\boldsymbol{x}$ and Tile $(\boldsymbol{\alpha}[i])=t$. For a window movie $M=$ $\left(v_{0}, g_{0}\right),\left(v_{1}, g_{1}\right), \ldots$, we write $M[k]$ to be the pair $\left(v_{k}, g_{k}\right)$ in the enumeration of $M$ and $\operatorname{Pos}(M[k])=v_{k}$, where $v_{k}$ is a vertex of a grid graph. 
We now proceed with the proof, throughout which we will assume that $M=$ $\mathcal{B}\left(M_{\boldsymbol{\alpha}, w}\right)$ and $M^{\prime}=\mathcal{B}\left(M_{\boldsymbol{\alpha}, w^{\prime}}\right)$. Since $M+\boldsymbol{c}=M^{\prime}$ for some $\boldsymbol{c} \neq(0,0)$ and $w$ and $w^{\prime}$ are both closed windows, it must be the case that the seed tile of $\alpha$ is in $\operatorname{dom} \alpha_{O} \cap \operatorname{dom} \alpha_{O}^{\prime}$ or in $\operatorname{dom} \alpha_{I} \cap \operatorname{dom} \alpha_{I}^{\prime}$. In other words, the seed tile cannot be in dom $\alpha_{I} \backslash$ dom $\alpha_{I}^{\prime}$ nor in dom $\alpha_{I}^{\prime} \backslash \operatorname{dom} \alpha_{I}$. Therefore, assume without loss of generality that the seed tile is in $\operatorname{dom} \alpha_{O} \cap \operatorname{dom} \alpha_{O}^{\prime}$.

The algorithm in Figure 1 describes how to produce a new valid assembly sequence $\gamma$.

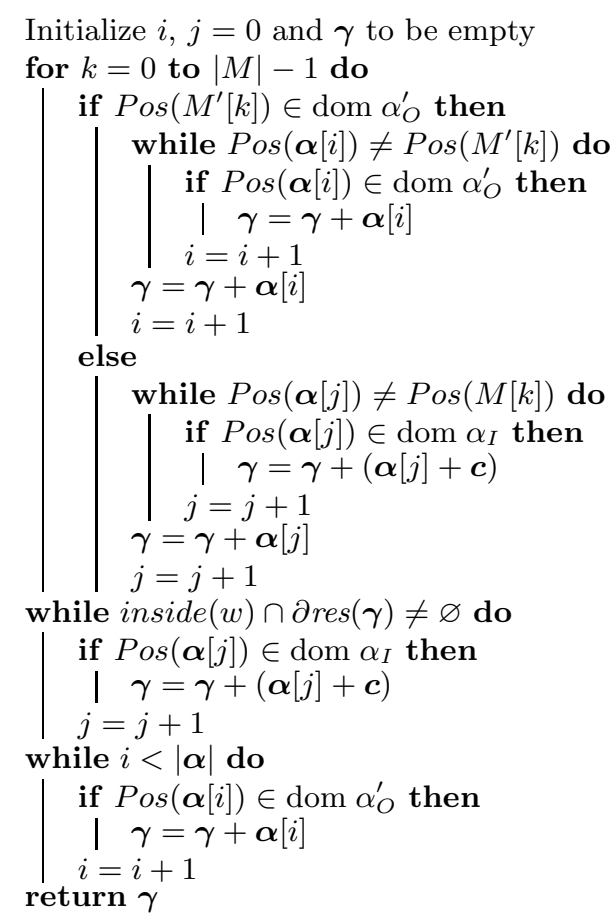

Fig. 1. The algorithm to produce a valid assembly sequence $\gamma$.

If we assume that the assembly sequence $\gamma$ ultimately produced by the algorithm is valid, then the result of $\gamma$ is indeed $\alpha_{O}^{\prime} \cup\left(\alpha_{I}+\boldsymbol{c}\right)$. Observe that $\alpha_{I}$ must be finite, which implies that $M$ is finite. If $|\boldsymbol{\alpha}|<\infty$, then all loops will terminate. If $|\boldsymbol{\alpha}|=\infty$, then $\left|\alpha_{O}^{\prime}\right|=\infty$ and the first two loops will terminate and the last loop will run forever. In either case, for every tile in $\alpha_{O}^{\prime}$ and $\alpha_{I}+\boldsymbol{c}$, the algorithm adds a step to the sequence $\gamma$ involving the addition of this tile to the assembly. However, we need to prove that the assembly sequence $\gamma$ is valid. It may be the case that either: 1 . there is insufficient bond strength between the tile to be placed and the existing neighboring tiles, or 2. a tile is already present at this location.

Case 1: In this case, we claim the following: at each step of the algorithm, the current version of $\gamma$ is a valid assembly sequence whose result is a producible 
subassembly of $\alpha_{O}^{\prime} \cup\left(\alpha_{I}+\boldsymbol{c}\right)$. Note that three loops in the algorithm iterate through all steps of $\boldsymbol{\alpha}$, such that at any time when adding $\boldsymbol{\alpha}[i]($ or $\boldsymbol{\alpha}[j]+\boldsymbol{c})$ to $\boldsymbol{\gamma}$, all steps of the window movie occurring before $\boldsymbol{\alpha}[i]$ (or $\boldsymbol{\alpha}[j]$ ) in $\boldsymbol{\alpha}$ have occurred. Similarly, all tiles in $\alpha_{O}^{\prime}$ (or $\alpha_{I}+\boldsymbol{c}$ ) added to $\alpha$ before step $i$ in the assembly sequence have occurred.

So, if the tile Tile $(\boldsymbol{\alpha}[i])$ that is added to the subassembly of $\alpha$ produced after $i-1$ steps can bond at a location in $\alpha_{O}^{\prime}$ to form a $\tau$-stable assembly, then the same tile added to the producible assembly of $\gamma$ must also bond to the same location in $\gamma$, as the neighboring glues consist of (i) an identical set of glues from tiles in the subassembly of $\alpha_{O}^{\prime}$ and (ii) glues on the side of the window movie containing $\alpha_{I}+\boldsymbol{c}$. Similarly, the tiles of $\alpha_{I}+\boldsymbol{c}$ must also be able to bind.

Case 2: Since we only assume that $\mathcal{B}\left(M_{\boldsymbol{\alpha}, w}\right)+\boldsymbol{c}=\mathcal{B}\left(M_{\boldsymbol{\alpha}, w^{\prime}}\right)$, as opposed to the stronger condition $\mathcal{B}\left(M_{\boldsymbol{\alpha}, w+\boldsymbol{c}}\right)=\mathcal{B}\left(M_{\boldsymbol{\alpha}, w^{\prime}}\right)$, which is assumed in the original WML, we must show that dom $\left(\alpha_{I}+\boldsymbol{c}\right) \cap \operatorname{dom} \alpha_{O}^{\prime}=\varnothing$. To see this, observe that, by assumption, $w+\boldsymbol{c}$ is enclosed in $w^{\prime}$, which, by definition, means that inside $(w+\boldsymbol{c}) \subseteq$ inside $\left(w^{\prime}\right)$. Then we have $\boldsymbol{x} \in \operatorname{dom} \alpha_{O}^{\prime} \Rightarrow \boldsymbol{x} \in$ outside $\left(w^{\prime}\right) \Rightarrow$ $\boldsymbol{x} \notin$ inside $\left(w^{\prime}\right) \Rightarrow \boldsymbol{x} \notin$ inside $(w+\boldsymbol{c}) \Rightarrow \boldsymbol{x} \notin \operatorname{dom}\left(\alpha_{I}+\boldsymbol{c}\right)$. Thus, locations in $\alpha_{I}+\boldsymbol{c}$ only have tiles from $\alpha_{I}$ placed in them, and locations in $\alpha_{O}^{\prime}$ only have tiles from $\alpha_{O}^{\prime}$ placed in them.

So the assembly sequence of $\boldsymbol{\gamma}$ is valid, i.e., every addition to $\gamma$ adds a tile to the assembly to form a new producible assembly. Since we have a valid assembly sequence, as argued above, the finished producible assembly is $\alpha_{O}^{\prime} \cup\left(\alpha_{I}+\boldsymbol{c}\right)$.

\section{Main result: scaled tree fractals do not strictly self-assemble}

In this section, we first define some notation and then prove our main result.

\subsection{Notation}

Recall that each stage $X_{s}(s>1)$ of a $g$-dssf (scaled by a factor $c$ ) is made up of copies of the previous stage $X_{s-1}$, each of which is a square of size $c g^{s-1}$. In the proof of our main result, we will need to refer to one of the squares of size $c g^{s-2}$ inside the copies of stage $X_{s-1}$, leading to the following notation.

Notation 6 Let $c \in \mathbb{Z}^{+}, 1<s \in \mathbb{N}$ and $1<g \in \mathbb{N}$. Let e, f, $p, q \in \mathbb{N}_{g}$. We use $S_{s}^{c}(e, f, p, q)$ to denote $\left\{0,1, \ldots, c g^{s-2}-1\right\}^{2}+c g^{s-1}(e, f)+c g^{s-2}(p, q)$ and $W_{s}^{c}(e, f, p, q)$ to denote the square-shaped, closed window whose inside is $S_{s}^{c}(e, f, p, q)$.

In Figure 2 below, the small and large red windows are $W_{2}^{1}(0,1,3,2)$ and $W_{3}^{1}(0,1,3,2)$, respectively.

Next, we will need to translate a small window to a position inside a larger window. These two windows will correspond to squares at the same relative position in different stages $i$ and $j$ of a $g$-dssf. 
Notation 7 Let $c \in \mathbb{Z}^{+}, i, j \in \mathbb{N} \backslash\{0,1\}$, with $i<j$, and $e, f, p, q \in \mathbb{N}_{g}$. We use $\boldsymbol{t}_{i \rightarrow j}^{c}(e, f, p, q)$ to denote the vector joining the southwest corner of $W_{i}^{c}(e, f, p, q)$ to the southwest corner of $W_{j}^{c}(e, f, p, q)$. In other words, $\boldsymbol{t}_{i \rightarrow j}^{c}(e, f, p, q)=$ $\left(c\left(g^{j-1}-g^{i-1}\right) e+c\left(g^{j-2}-g^{i-2}\right) p, c\left(g^{j-1}-g^{i-1}\right) f+c\left(g^{j-2}-g^{i-2}\right) q\right)$.

For example, in Figure 2 below, $\boldsymbol{t}_{2 \rightarrow 3}^{1}(0,1,3,2)=(9,18)$.

Finally, to apply Lemma 1 we will need the bond-forming submovies to line up. Therefore, once the two square windows share their southwest corner after using the translation defined above, we will need to further translate the smallest one either up or to the right, or both, depending on which side of the windows contains the bond-forming glues, which, in the case of scaled tree fractals, always form a straight (vertical or horizontal) line of length $c$. We will compute the coordinates of this second translation in our main proof. For now, we establish an upper bound on these coordinates that will ensure that the translated window will remain enclosed in the larger window.

Lemma 2. Let $c \in \mathbb{Z}^{+}, i, j \in \mathbb{N} \backslash\{0,1\}$, with $i<j, e, f, p, q \in \mathbb{N}_{g}$, and $x, y \in \mathbb{N}$. Let $m=c\left(g^{j-2}-g^{i-2}\right)$. If $x \leq m$ and $y \leq m$, then the window $W_{i}^{c}(e, f, p, q)+$ $\boldsymbol{t}_{i \rightarrow j}^{c}(e, f, p, q)+(x, y)$ is enclosed in the window $W_{j}^{c}(e, f, p, q)$.

Finally, the following lemma establishes that any scaled tree fractal $\mathbf{T}^{c}$ contains an infinite number of closed windows that all cut the fractal along a single line of glues.

Lemma 3. Let $\mathbf{T}$ be any tree fractal with generator $G$. If $c \in \mathbb{Z}^{+}$, then it is always possible to pick one pier $(p, q)$ and one point $(e, f)$, both in $G$, such that, for $1<s \in \mathbb{N}, W_{s}^{c}(e, f, p, q)$ encloses a configuration that is connected to $\mathbf{T}^{c}$ via a single connected (horizontal or vertical) line of glues of length $c$.

The proofs of the lemmas in this sub-section are omitted from this version of the paper due to lack of space.

\subsection{Application to scaled tree fractals}

The main contribution of this paper is the following theorem.

Theorem 2. Let $\mathbf{T}$ be any tree fractal. If $c \in \mathbb{Z}^{+}$, then $\mathbf{T}^{c}$ does not strictly self-assemble in the aTAM.

Proof. Let $\mathbf{T}$ be any tree fractal with a $g \times g$ generator $G$, where $1<g \in \mathbb{N}$. Let $c$ be any positive integer. For the sake of obtaining a contradiction, assume that $\mathbf{T}^{c}$ does strictly self-assemble in some TAS $\mathcal{T}=(T, \sigma, \tau)$. Further assume that $\boldsymbol{\alpha}$ is some assembly sequence in $\mathcal{T}$ whose result is $\alpha$, such that $\operatorname{dom} \alpha=\mathbf{T}^{c}$.

According to Lemma 3, we can always pick one pier $(p, q)$ and a point $(e, f)$, both in $G$, such that, for $1<s \in \mathbb{N}$, the window $W_{s}^{c}(e, f, p, q)$, which we will abbreviate $w_{s}$, encloses a configuration that is connected to $\mathbf{T}^{c}$ via a single 


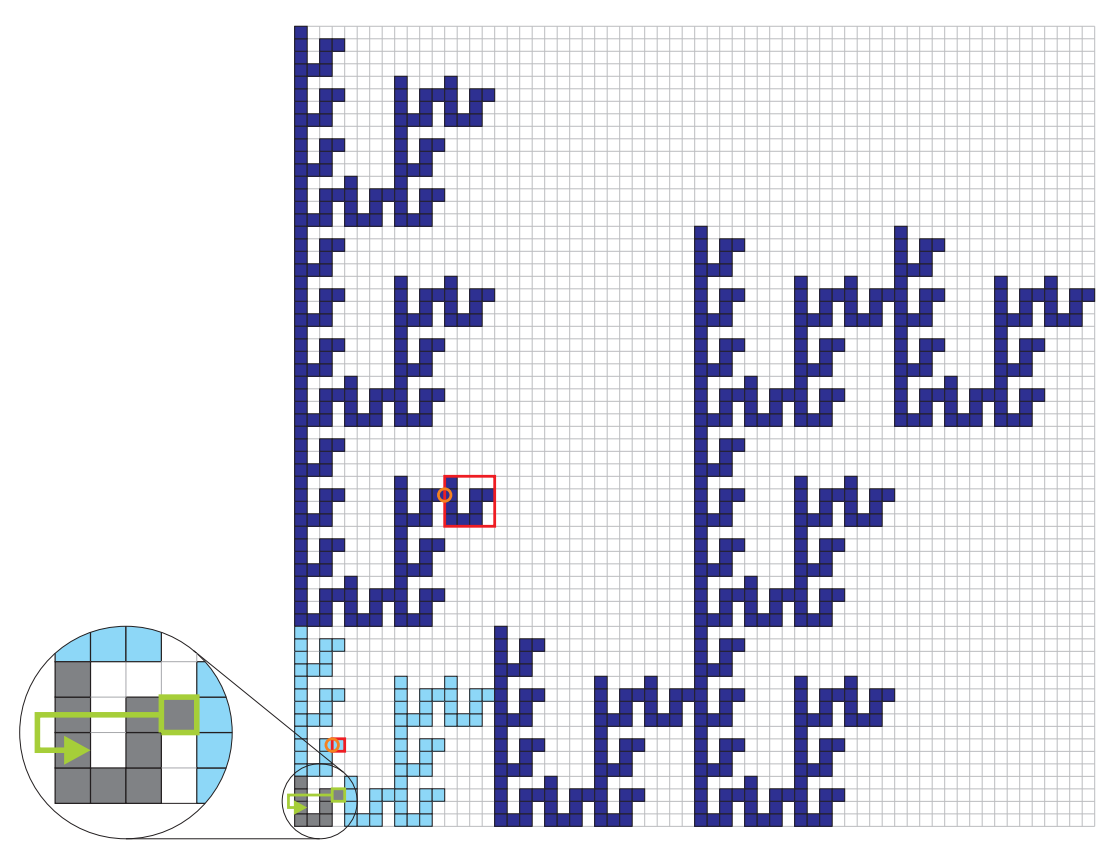

Fig. 2. First three stages $(s=1,2,3)$ of an unscaled $(c=1)$ 4-dssf tree fractal with an east-pointing pier at position $(3,2)$ (the green square). The $E$-free point $(0,1)$ is at the tip of the green arrow. In other words, $(p, q)=(3,2)$, and $(e, f)=(0,1)$.

line of glues of length $c 3$ The maximum number of distinct combinations and orderings of glue positionings along this line of glues is finite 4 By the generalized pigeonhole principle, since $\left|\left\{w_{s} \mid 1<s \in \mathbb{N}\right\}\right|$ is infinite, there must be at least one bond-forming submovie such that an infinite number of windows generate this submovie (up to translation). Let us pick two such windows, say, $w_{i}$ and $w_{j}$ with $i<j$, such that $\mathcal{B}\left(M_{\boldsymbol{\alpha}, w_{i}}\right)$ and $\mathcal{B}\left(M_{\boldsymbol{\alpha}, w_{j}}\right)$ are equal (up to translation). We must pick these windows carefully, since as stated in the proof of Lemma 1, the seed of $\alpha$ must be either in both windows or in neither. This condition can always be satisfied. The only case where the seed is in more than one window is when it is at position $(0,0)$ and $e=f=p=q=0$, which implies that all windows include the origin. In all other cases, none of the windows overlap. So, if the seed belongs to one of them, say $w_{k}$, then we can pick any $i$ greater than $k$ (and $j>i$ ). Finally, if the seed does not belong to any windows, then any choice of $i$ and $j>i$ will do.

\footnotetext{
${ }^{3}$ Without loss of generality, we will assume that this line of glues is positioned on the western side of the windows and is thus vertical (see the orange circles in Figure 2 , where $s=2$ and $s=3$ for the small and large red windows, respectively, and $(p, q)=(3,2)$ and $(e, f)=(0,1))$, because the chosen pier in our example points east. A similar reasoning holds for piers pointing north, south or west.

${ }^{4}$ This number is $\left(T_{\text {glue }}\right)^{2 c} \cdot(2 c)$ !, where $T_{\text {glue }}$ is the total number of distinct glue types in $T$.
} 


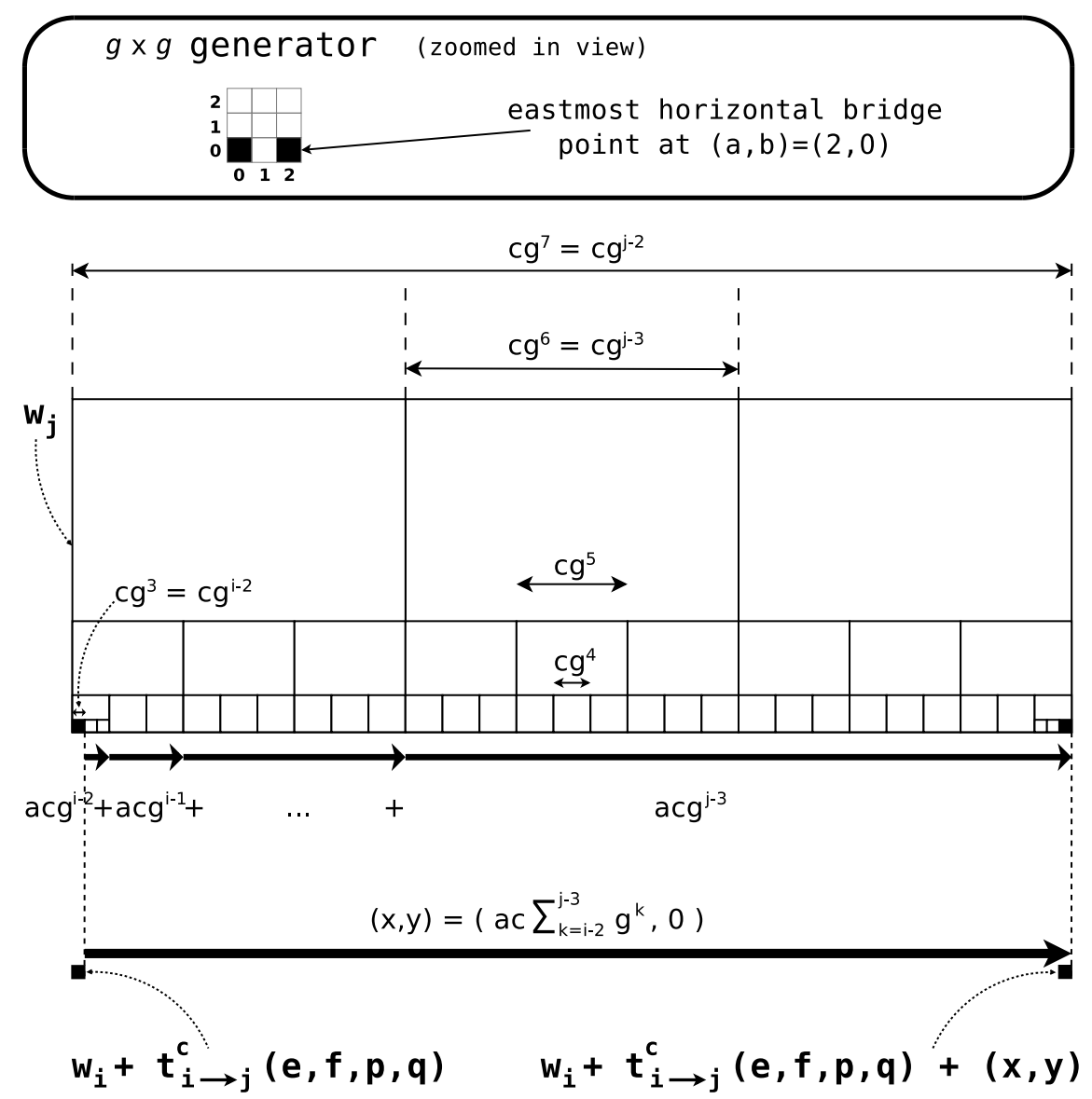

Fig. 3. $(x, y)$ translation needed to align $w_{i}$ and $w_{j}$ on their east side once their southwest corners already match. Example with a west-pointing pier (not shown) and $g=3$, $i=5, j=9,(a, b)=(2,0)$.

We will now prove that $w_{i}$ and $w_{j}$ satisfy the two conditions of Lemma 1

First, we compute $\boldsymbol{c}$ such that $\mathcal{B}\left(M_{\boldsymbol{\alpha}, w_{i}}\right)+\boldsymbol{c}=\mathcal{B}\left(M_{\boldsymbol{\alpha}, w_{j}}\right)$. We know that $w_{i}+\boldsymbol{t}_{i \rightarrow j}^{c}(e, f, p, q)$ and $w_{j}$ share their southwest corner. We need to perform one more translation to align the bond-forming glues of $w_{i}$ and $w_{j}$. We use $(a, b)$ to denote the position of the western point in the horizontal bridge of the generator. In our example (east-pointing pier), $a=0$ and $b$ is a variable with domain $\mathbb{N}_{g}\left(b=2\right.$ in Figure 2). To align the bond-forming glues of $w_{i}$ and $w_{j}$, we must translate $w_{i}+\boldsymbol{t}_{i \rightarrow j}^{c}(e, f, p, q)$ by $(x, y)=\left(0, b c \sum_{k=i-2}^{j-3} g^{k}\right)$. The general computation for this translation is illustrated in Figure 3 . Since $x \leq m$ (as defined in Lemma 21) and $b c \sum_{k=i-2}^{j-3} g^{k} \leq(g-1) c \sum_{k=i-2}^{j-3} g^{k}=$ $c\left(\sum_{k=i-1}^{j-2} g^{k}-\sum_{k=i-2}^{j-3} g^{k}\right)=c\left(g^{j-2}-g^{i-2}\right)=m$, we can apply Lemma 2 to 
infer that, with $\boldsymbol{c}=\boldsymbol{t}_{i \rightarrow j}^{c}(e, f, p, q)+(x, y), w_{i}+\boldsymbol{c}$ is enclosed in $w_{j}$. Therefore, the second condition of Lemma 1 holds.

Second, by construction, $\mathcal{B}\left(M_{\boldsymbol{\alpha}, w_{i}}\right)+\boldsymbol{c}=\mathcal{B}\left(M_{\boldsymbol{\alpha}, w_{j}}\right)$. Therefore, the first condition of Lemma 1 holds.

In conclusion, the two conditions of Lemma 1 are satisfied, with $\alpha_{I}$ and $\alpha_{O}^{\prime}$ defined as the intersection of $\mathbf{T}^{c}$ with the inside of $w_{i}$ and the outside of $w_{j}$, respectively. We can thus conclude that the assembly $\alpha_{I} \cup\left(\alpha_{O}^{\prime}-\boldsymbol{c}\right)$ is producible in $\mathcal{T}$. Note that this assembly is identical (up to translation) to $\mathbf{T}^{c}$, except that the interior of the large window $w_{j}$ is replaced by the interior of the small window $w_{i}$. Since the configurations in these two windows cannot be identical, we have proved that $\mathcal{T}$ does not strictly self-assemble $\mathbf{T}^{c}$, which is a contradiction.

\section{Conclusion}

In this paper, we made three contributions. First, we gave a new characterization of tree fractals in terms of simple geometric properties of their generator. Second, we proved a new variant of the Window Movie Lemma in [5], which we call the "Closed Window Movie Lemma." Third, we proved that no scaled-up version of any discrete self-similar tree fractal strictly self-assembles in the aTAM. In future work, we plan to extend this result to larger classes of non-tree fractals similar to the class of pinch-point fractals in [6].

\section{References}

1. Steven M. Kautz and James I. Lathrop, Self-assembly of the discrete Sierpinski carpet and related fractals, DNA, 2009, pp. 78-87.

2. Steven M. Kautz and Brad Shutters, Self-assembling rulers for approximating generalized sierpinski carpets, Algorithmica 67 (2013), no. 2, 207-233.

3. James I. Lathrop, Jack H. Lutz, and Scott M. Summers, Strict self-assembly of discrete Sierpinski triangles, Theoretical Computer Science 410 (2009), 384-405.

4. Jack H. Lutz and Brad Shutters, Approximate self-assembly of the sierpinski triangle, Theory Comput. Syst. 51 (2012), no. 3, 372-400.

5. P.-E. Meunier, M. J. Patitz, S. M. Summers, G. Theyssier, A. Winslow, and D. Woods, Intrinsic universality in tile self-assembly requires cooperation, Proceedings of the 25th Annual ACM-SIAM Symposium on Discrete Algorithms (SODA), 2014, pp. $752-771$.

6. Matthew J. Patitz and Scott M. Summers, Self-assembly of discrete self-similar fractals, Natural Computing 1 (2010), 135-172.

7. Paul W. K. Rothemund, Theory and experiments in algorithmic self-assembly, Ph.D. thesis, University of Southern California, December 2001.

8. Nadrian C. Seeman, Nucleic-acid junctions and lattices, Journal of Theoretical Biology 99 (1982), 237-247.

9. nal of Biomolecular Structural Dynamics 8 (1990), 573-581.

10. Hao Wang, Proving theorems by pattern recognition - II, The Bell System Technical Journal XL (1961), no. 1, 1-41.

11. Erik Winfree, Algorithmic self-assembly of DNA, Ph.D. thesis, California Institute of Technology, June 1998. 